\title{
AVALIAÇÃO DAS PROPRIEDADES FÍSICO-MECÂNICAS DE PAINÉIS COMPENSADOS DE Toona ciliata M. Roem. var. australis
}

\author{
Vanessa Cristina do Sacramento Albinoํㅡ, Vânia Aparecida de Sá2, Lina Bufalino², \\ Lourival Marin Mendes ${ }^{3}$, Natalia Amarante Almeida ${ }^{4}$
}

(recebido: 28 de setembro de 2009; aceito: 30 de novembro de 2010)

\begin{abstract}
RESUMO: Objetivou-se, com o presente trabalho, avaliar as propriedades físicas e mecânicas de painéis compensados produzidos a partir de madeira de Toona ciliata com duas pressões e duas gramaturas distintas, além da influência da inclusão de lâminas de Pinus sp. na qualidade desses painéis. Foram produzidos compensados de cinco lâminas com resina fenol-formaldeído. Os ensaios físico-mecânicos foram realizados de acordo com as especificações descritas na norma ABNT 31:000.05-001/2(2001). Os resultados de densidade aparente, absorção de água após 24 horas, flexão estática (MOR e MOE) e resistência ao cisalhamento seco na linha de cola apresentaram diferenças significativas entre os tratamentos. Todos os tratamentos apresentaram valor de MOE e MOR paralelo acima do exigido pela norma NBR 31.000.001/2:2001, no entanto apenas os painéis com inclusão de Pinus sp. no miolo atingiram MOE e MOR perpendicular acima do estipulado pela mesma. Para os ensaios de resistência de colagem ao esforço do cisalhamento, os painéis apresentaram valores superiores ao mínimo exigido pela norma EN 314-2 (1993). Comparando-se os painéis produzidos somente com Toona ciliata, não foi observada influência da gramatura de cola e da pressão sobre nenhuma das propriedades físicas e mecânicas avaliadas. A inclusão de lâminas de Pinus sp. tanto nas camadas internas quanto nas camadas externas dos painéis contribuiu para melhorar a maioria das propriedades físicas e mecânicas.
\end{abstract}

Palavras-chave: Compensado, cedro australiano, propriedades físico-mecânicas.

\section{EVALUATION OF THE PHYSICAL AND MECHANICAL PROPERTIES OF PLYWOODS PRODUCED WITH Toona ciliata M. Roem. var. australis}

\begin{abstract}
The aim of this work was to evaluate the physical and mechanical properties of plywood panels produced with Toona ciliata wood using two different pressure levels and two spread rates; besides the effect of Pinus sp. veneer inclusion on the quality of the panels. The phenol formaldehyde resin was used to produce plywood of five veneers. The physical-mechanical tests were conducted according to ABNT 31:000.05-001/2:2001 standard. The results of apparent density, water absorption after 24 hours, static bending (MOR and MOE) and glue line dry shear strength presented significant statistical differences between the treatments. All the treatments presented parallel MOE and MOR value above the one established by the NBR 31.000.001/2:2001 standard; however just the panels with inclusion of Pinus sp. in the inner presented perpendicular MOE and MOR above the one established by the same. For the glue line shear strength tests, the panels presented values above the minimum established by EN 314-2 (1993) standard.It was not observed influence of the spread rate and pressure on the physical and mechanical properties between the panels produced only with Toona ciliata. The inclusion of Pinus sp. veneers both in the face and inner of the panels contributed to improve most of the physical and mechanical properties.
\end{abstract}

Key words: Plywood, red Australian cedar, physical-mechanical properties.

\section{INTRODUÇÃO}

A indústria de painéis de madeira tem uma acentuada importância para a economia brasileira devido à geração de divisas e empregos, especialmente nos setores moveleiro e de construção civil.

Dentre os diversos tipos de painéis produzidos, destacam-se os compensados, cujo princípio, segundo
Iwakiri (2005), é baseado na sobreposição cruzada de lâminas em número ímpar de camadas, com a direção da grã perpendicular entre as camadas adjacentes.

Os painéis compensados podem ser classificados como de uso exterior e interior, diferenciados principalmente em função do tipo de resina utilizada. O compensado de uso exterior é definido como painel produzido com adesivo à prova d'água, apresentando características de

\footnotetext{
${ }^{1}$ Engenheira Florestal, Mestre em Ciência e Tecnologia da Madeira - Departamento de Ciências Florestais - Universidade Federal de Lavras Cx. P. 3037 - 37200-000 - Lavras, MG - vanessa.floresta@hotmail.com

${ }^{2}$ Engenheira Florestal, Doutoranda em Ciência e Tecnologia da Madeira - Departamento de Ciências Florestais - Universidade Federal de Lavras Cx. P. 3037 -37200-000 - Lavras, MG - vania_desa@yahoo.com.br; linabufalino@yahoo.com.br

${ }^{3}$ Engenheiro Florestal, Professor Doutor em Engenharia Florestal - Departamento de Ciências Florestais - Universidade Federal de Lavras - Cx. P. 3037 37200-000 - Lavras, MG - lourival@dcf.ufla.br

${ }^{4}$ Mestre em Ciência e Tecnologia da Madeira - Departamento de Ciências Florestais - Universidade Federal de Lavras - Cx. P. 3037 - $37200-000$ Lavras, MG - natamarant@gmail.com
} 
alta resistência mecânica e destinado a aplicações que requerem alta resistência à umidade do ambiente e ao contato direto com a água (ASSOCIAÇÃO BRASILEIRA DE NORMAS TÉCNICAS - ABNT, 2001). Esses produtos são empregados na indústria moveleira, construção civil, embalagens e construção naval.

Com relação ao processo de produção de compensado, as interações entre as variáveis do mesmo são muito abrangentes. As características inerentes à madeira, o tipo, a quantidade e a composição do adesivo, assim como os procedimentos empregados na colagem de lâminas, são de fundamental importância na qualidade dos painéis (BALDWIN, 1995; SELLERS, 1985).

Na década de 40, o pinheiro brasileiro (Araucaria angustifolia) era a principal espécie utilizada para a produção de compensados no Brasil. Já na década de 70 , com a escassez dessa espécie, as madeiras tropicais passaram a ser a principal matéria-prima para as indústrias de compensados (ASSOCIAÇÃO BRASILEIRA DAS INDÚSTRIAS DE MADEIRA PROCESSADA MECANICAMENTE - ABIMCI, 2001; IWAKIRI; PRATA, 2008). No entanto, a partir da década de 90, em consequência da crescente pressão ambiental sobre a exploração de florestas tropicais, quantidades expressivas de madeiras de pinus, provenientes de reflorestamentos, passaram a ser empregadas para esse fim (IWAKIRI et al., 2006).

Uma nova alternativa para produção de compensados é o cedro australiano (Toona ciliata var. australis - Meliaceae), que vem ganhando destaque junto aos produtores que estão buscando diversificar suas atividades a fim de aumentar sua fonte de renda. É uma espécie exótica proveniente de várias regiões da Austrália. Apresenta-se como de grande porte, podendo alcançar cerca de $50 \mathrm{~m}$ de altura e um diâmetro de 1,5 metros ou mais. Sua madeira, de uso nobre, é indicada para a fabricação de móveis finos e acabamentos na construção civil. No Brasil, especialmente na região sudeste, essa espécie encontrou condições favoráveis ao seu desenvolvimento (LAMPRECHT, 1990; PINHEIRO et al., 2006).

Assim, com o presente trabalho, objetivouse avaliar as propriedades físicas e mecânicas de compensados produzidos a partir de madeira de Toona ciliata com duas pressões e duas gramaturas distintas, além da influência da inclusão de lâminas de Pinus sp. na qualidade desses painéis.

Cerne, Lavras, v. 17, n. 1, p. 103-108, jan./mar. 2011

\section{MATERIAL E MÉTODOS}

Neste trabalho foram utilizadas madeiras de Toona ciliata M. Roem. var australis (18 anos e densidade básica de $0,32 \mathrm{~g} / \mathrm{cm}^{3}$ ) e Pinus sp. (30 anos e densidade básica de $0,5 \mathrm{~g} / \mathrm{cm}^{3}$ ) procedentes, respectivamente, de um plantio comercial localizado no município de Marechal Floriano - ES, e de um plantio experimental do campus da Universidade Federal de Lavras, Lavras - MG.

As toras foram previamente aquecidas, em tanque d'água à temperatura de $65^{\circ} \mathrm{C}$, durante 60 horas, para obtenção das lâminas em torno laminador com espessura nominal de $2 \mathrm{~mm}$. As lâminas foram guilhotinadas nas dimensões finais de $480 \mathrm{~mm} \times 480 \mathrm{~mm}$ e posteriormente tabicadas no plano horizontal para secagem natural em local coberto até atingirem a umidade de equilíbrio higroscópico. Em seguida, o material teve seu teor de umidade reduzido a $8 \%$ em estufa com circulação forçada de ar. Classificaram-se as lâminas de forma a separar as de melhor qualidade para compor a capa dos painéis.

Para formação dos painéis compensados, foram utilizadas cinco lâminas dispostas de forma cruzada em relação à direção da grã, coladas com adesivo fenolformaldeído com pH de 11,8, teor de sólidos de 50,37\%, viscosidade de $4.200 \mathrm{cP}$. O tempo de assemblagem foi de 15 minutos, enquanto que a temperatura e tempo de prensagem foram, respectivamente, $150{ }^{\circ} \mathrm{C}$ e 10 minutos.

$\mathrm{O}$ delineamento experimental foi inteiramente casualizado composto por 6 tratamentos e 3 repetições, totalizando 18 painéis (Tabela 1). Foram avaliadas duas gramaturas de cola e duas pressões nos painéis de Toona ciliata. Além disso, avaliou-se a combinação das lâminas dessa espécie com lâminas de Pinus sp. na capa e no miolo dos painéis.

Tabela 1 - Plano experimental.

Table 1 - Experimental design used.

\begin{tabular}{cccc}
\hline Tratamento & Composição & $\begin{array}{c}\text { Gramatura } \\
\left(\mathrm{g} / \mathrm{m}^{2}\right)\end{array}$ & $\begin{array}{c}\text { Pressão } \\
\left(\mathrm{kgf} / \mathrm{cm}^{2}\right)\end{array}$ \\
\hline 1 & $\mathrm{~T}$ & 320 & 8 \\
2 & $\mathrm{~T}$ & 320 & 5 \\
3 & $\mathrm{~T}$ & 280 & 8 \\
4 & $\mathrm{~T}$ & 280 & 5 \\
5 & $\mathrm{P} / \mathrm{T}$ & 320 & 8 \\
6 & $\mathrm{~T} / \mathrm{P}$ & 320 & 8
\end{tabular}

T: Toona ciliata M. Roem.; P/T: Pinus sp. na face e Toona ciliata M. Roem. no miolo; T/P: Toona ciliata M. Roem. na face e Pinus sp. no miolo. 
Os painéis foram acondicionados em câmara climática com umidade relativa de $65 \pm 3 \%$ e temperatura de $20 \pm 1{ }^{\circ} \mathrm{C}$. Em seguida, retiraram-se os corpos de prova para realização de ensaios de resistência da linha de cola aos esforços de cisalhamento pelo teste seco, úmido e pósfervura, flexão estática paralela e perpendicular (módulos de elasticidade e ruptura), densidade, teor de umidade e absorção de água. Os ensaios foram realizados de acordo com os procedimentos descritos na norma ABNT (2001).

A análise estatística dos resultados foi realizada pela análise de variância e as médias dos tratamentos foram comparadas pelo Teste de Tukey, a $95 \%$ de probabilidade.

\section{RESULTADOS E DISCUSSÃO}

\subsection{Propriedades físicas dos compensados}

Os valores médios da densidade aparente, teor de umidade e absorção de água dos compensados estão apresentados na Tabela 2.

Tabela 2 - Valores médios das propriedades físicas dos painéis compensados.

Table 2 - Average values of the plywood panels physical properties.

\begin{tabular}{lccc}
\hline Tratamento & $\begin{array}{c}\text { Densidade } \\
\text { aparente }\left(\mathrm{g} / \mathrm{cm}^{3}\right)\end{array}$ & $\begin{array}{c}\text { Teor de } \\
\text { umidade }(\%)\end{array}$ & $\begin{array}{c}\text { Absorção de } \\
\text { água }(\%)\end{array}$ \\
\hline $1\left(\mathrm{~T}_{320 / 8}\right)$ & $0,44 \mathrm{~b}$ & $9,90 \mathrm{a}$ & $74,67 \mathrm{ab}$ \\
$2\left(\mathrm{~T}_{320 / 5}\right)$ & $0,42 \mathrm{~b}$ & $10,13 \mathrm{a}$ & $84,67 \mathrm{a}$ \\
$3\left(\mathrm{~T}_{280 / 8}\right)$ & $0,44 \mathrm{~b}$ & $9,87 \mathrm{a}$ & $81,00 \mathrm{a}$ \\
$4\left(\mathrm{~T}_{280 / 5}\right)$ & $0,43 \mathrm{~b}$ & $10,37 \mathrm{a}$ & $82,67 \mathrm{a}$ \\
$5\left(\mathrm{P} / \mathrm{T}_{320 / 8}\right)$ & $0,55 \mathrm{a}$ & $10,07 \mathrm{a}$ & $56,67 \mathrm{~b}$ \\
$6\left(\mathrm{~T} / \mathrm{P}_{320 / 8}\right)$ & $0,54 \mathrm{a}$ & $10,30 \mathrm{a}$ & $73,02 \mathrm{ab}$ \\
\hline $\mathrm{F}$ calculado & $16,416^{*}$ & $0,150^{\text {ns }}$ & $4,475^{*}$ \\
$\mathrm{CV}(\%)$ & 5,27 & 9,02 & 11,14 \\
\hline
\end{tabular}

T: Toona ciliata M. Roem.; P/T: Pinus sp. na face e Toona ciliata M. Roem. no miolo; T/P: Toona ciliata M. Roem. na face e Pinus sp. no miolo; gramatura de cola: 320 ou $280 \mathrm{~g} / \mathrm{m}^{2}$; pressão: 5 ou $8 \mathrm{kgf} / \mathrm{cm}^{2}$.

$\mathrm{e}^{*}$ : valores de F significativo a 5\% de significância; $\mathrm{e}^{\text {ns: }}$ : valor de F não significativo a 5\% de significância.

Médias seguidas pela mesma letra não diferem estatisticamente entre si pelo teste de Tukey a 95\% de probabilidade.

Pode-se constatar que houve efeito significativo dos tratamentos em relação à densidade aparente e que os painéis produzidos com lâminas de Pinus sp. tanto na face quanto no miolo apresentaram valor médio desta propriedade estatisticamente superior em relação aos painéis produzidos somente com lâminas de Toona ciliata. Isso possivelmente ocorreu devido à maior densidade básica da madeira de Pinus sp. em relação à da Toona ciliata. Segundo Kollmann et al. (1975), a densidade aparente do compensado é cerca de 5\% maior em relação à madeira correspondente, quando não são aplicadas pressões elevadas, e depende da espécie da madeira, da umidade das lâminas e das variáveis do ciclo de prensagem, como temperatura e pressão.

Para teor de umidade dos painéis compensados, não houve efeito significativo de nenhum dos tratamentos sobre a mesma. Tal resultado pode indicar que o período de climatização dos painéis antes dos ensaios foi adequado, tornando os seus teores de umidade uniformes.

Em relação à absorção de água, é possível observar que houve efeito significativo dos tratamentos sobre a mesma. De forma geral, os tratamentos que mais absorveram água foram aqueles cujos painéis foram compostos somente por lâminas de Toona ciliata (T2, T3 e T4). Guimarães Júnior et al. (2009) mencionam que espécies de menor densidade apresentam maior potencial de absorção de água, devido à maior porosidade da madeira que permite ocupação com uma maior quantidade de água livre em relação a um material que apresenta densidade mais alta. No entanto, para este trabalho, quando se aplicou uma mesma pressão e gramatura, os painéis compostos por madeira de menor densidade, (T. ciliata - T1), e os painéis mistos (T6) apresentaram desempenho igual aos painéis com Pinus sp. na face (T5). Dessa forma, esses painéis foram mais resistentes para essa propriedade em relação aos demais e, portanto, teriam melhor desempenho para uso exterior.

\subsection{Propriedades mecânicas dos compensados}

Os valores médios para módulos de ruptura e elasticidade em flexão estática, nos sentidos perpendicular $(\perp)$ e paralelo $(/ /)$ às fibras, encontram-se na Tabela 3.

Os valores médios de MOR e MOE perpendicular às fibras variaram de 221,99 a $351,18 \mathrm{~kg} / \mathrm{cm}^{2}$ e de 10184,26 a $31844,16 \mathrm{~kg} / \mathrm{cm}^{2}$, respectivamente.

A maior média, em termos absolutos, de MOR e MOE perpendicular foi apresentada pelos painéis com inclusão de lâminas de Pinus sp. (T6) no miolo. Isso provavelmente ocorreu porque, neste tratamento, há duas lâminas de Pinus sp. na posição longitudinal em relação à direção de flexionamento, quando os corpos-de-prova são cortados com a grã da lâmina da capa orientada transversalmente em relação ao comprimento do corpo-

Cerne, Lavras, v. 17, n. 1, p. 103-108, jan./mar. 2011 
Tabela 3 - Valores médios de módulo de ruptura (MOR) e módulo de elasticidade (MOE) em flexão estática, nos sentidos perpendicular $(\perp)$ e paralelo $(/ /)$ às fibras.

Table 3 - Average values of rupture modulus (MOR) and elasticity modulus (MOE) for static bending, through parallel and cross grain direction.

\begin{tabular}{|c|c|c|c|c|}
\hline \multirow{2}{*}{ Tratamento } & \multicolumn{2}{|c|}{ Flexão estática $\perp$} & \multicolumn{2}{|c|}{ Flexão estática // } \\
\hline & $\mathrm{MOR}_{\perp}$ & $\mathrm{MOE}_{\perp}$ & $\mathrm{MOR} / /$ & $\mathrm{MOE} / /$ \\
\hline & 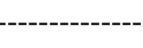 & --------------- & -----------. & ---------------. \\
\hline $1\left(\mathrm{~T}_{320 / 8}\right)$ & $221,99 \mathrm{~b}$ & $22921,04 \mathrm{ab}$ & $535,69 \mathrm{~b}$ & $78409,90 \mathrm{~b}$ \\
\hline $2\left(\mathrm{~T}_{320 / 5}\right)$ & $257,11 \mathrm{ab}$ & $23645,27 \mathrm{ab}$ & $530,43 \mathrm{~b}$ & $80573,86 \mathrm{~b}$ \\
\hline $3\left(\mathrm{~T}_{280 / 8}\right)$ & $243,07 \mathrm{~b}$ & 10184,26 b & $519,88 \mathrm{~b}$ & $78847,66 \mathrm{~b}$ \\
\hline $4\left(\mathrm{~T}_{280 / 5}\right)$ & $225,49 \mathrm{~b}$ & $20574,96 \mathrm{ab}$ & $538,51 \mathrm{~b}$ & $79109,86 \mathrm{~b}$ \\
\hline $5\left(\mathrm{P} / \mathrm{T}_{320 / 8}\right)$ & $292,52 \mathrm{ab}$ & $24166,51 \mathrm{ab}$ & $855,52 \mathrm{a}$ & $136436,90 \mathrm{a}$ \\
\hline $6\left(\mathrm{~T} / \mathrm{P}_{320 / 8}\right)$ & $351,18 \mathrm{a}$ & $31844,16 \mathrm{a}$ & $537,20 \mathrm{~b}$ & $81027,27 \mathrm{~b}$ \\
\hline F calculado & $4,755^{*}$ & $4,008^{*}$ & $53,838^{*}$ & $172,671^{*}$ \\
\hline $\mathrm{CV}(\%)$ & 14,76 & 27,34 & 5,32 & 3,44 \\
\hline
\end{tabular}

T: Toona ciliata M. Roem.; P/T: Pinus sp. na face e Toona ciliata M. Roem. no miolo; T/P: Toona ciliata M. Roem. na face e Pinus sp. no miolo; gramatura de cola: 320 ou $280 \mathrm{~g} / \mathrm{m}^{2}$; pressão: 5 ou $8 \mathrm{kgf} / \mathrm{cm}^{2}$.

$\mathrm{e}^{*}$ : valores de F significativo a 5\% de significância; $\mathrm{e}^{\text {ns: }}$ : valor de F não significativo a 5\% de significância.

Médias seguidas pela mesma letra não diferem estatisticamente entre si pelo teste de Tukey a $95 \%$ de probabilidade.

de-prova. Quanto aos tratamentos compostos somente por T. ciliata, não houve diferença estatística significativa para os valores de MOE e MOR perpendiculares. Para MOE, o tratamento 2 não apresentou diferença estatística significativa em relação aos painéis com inclusão de lâminas de Pinus sp. Já para os valores de MOE, o tratamento 2 apresentou-se estatisticamente inferior em relação ao tratamento 6 .

A norma NBR 31.000.001/2(2001) estabelece valores médios de MOE perpendicular para compensado do tipo forma de concreto (FOR), com a utilização de cinco lâminas na sua constituição, de no mínimo $25.493 \mathrm{kgf} / \mathrm{cm}^{2}$, enquanto que para MOR perpendicular, o valor mínimo desta propriedade deve ser de $306 \mathrm{kgf} / \mathrm{cm}^{2}$. Desse modo, tanto para $\mathrm{MOE}$ quanto para MOR, somente o tratamento 6 atingiu o mínimo estabelecido pela norma.

Os valores médios de MOR e MOE paralelo às fibras variaram de 519,88 a $855,52 \mathrm{~kg} / \mathrm{cm}^{2}$ e de 78409,9 a $136436,9 \mathrm{~kg} / \mathrm{cm}^{2}$, respectivamente, sendo que os painéis compostos por lâminas de Pinus sp. na face (T5) foram estatisticamente superiores em relação aos demais tratamentos. Isso provavelmente ocorreu porque neste tratamento há três lâminas de Pinus sp. na posição longitudinal em relação à direção de flexionamento, quando os corpos-de-prova são cortados com a grã da lâmina da capa orientada longitudinalmente em relação ao comprimento do corpo-de-prova.

Isso provavelmente ocorreu porque neste tratamento há maior número de lâminas (3) de Pinus sp. com a grã orientada longitudinalmente quando os corpos de prova são cortados na posição longitudinal em relação às duas lâminas da face. Sendo assim, a maior densidade do Pinus sp. contribui, efetivamente, para o aumento dessa propriedade mecânica. As médias dos tratamentos 1 a 4 (compostos somente por Toona ciliata) foram estatisticamente iguais, ou seja, a gramatura de cola e a pressão utilizada não exerceram efeito sobre esta propriedade física.

A norma NBR 31.000.001/2:2001 estabelece valores de MOE paralelo para compensado do tipo forma de concreto (FOR), com a utilização de cinco lâminas na sua constituição, de no mínimo $51.000 \mathrm{kgf} / \mathrm{cm}^{2}$, enquanto que para MOR paralelo, o valor mínimo desta propriedade deve ser $459 \mathrm{kgf} / \mathrm{cm}^{2}$. Desse modo, todos os tratamentos atingiram o mínimo estabelecido pela norma.

Iwakiri et al. (2006) avaliaram as propriedades mecânicas de compensados fenólicos de Eucaliptus grandis e de Pinus taeda e encontraram, para este último, valores médios de $105.969 \mathrm{kgf} / \mathrm{cm}^{2}$ para MOE e $732 \mathrm{kgf} / \mathrm{cm}^{2}$ para MOR, em flexão estática no sentido paralelo, ou seja,

Cerne, Lavras, v. 17, n. 1, p. 103-108, jan./mar. 2011 
inferiores ao tratamento 5 , referente aos painéis compostos por Pinus sp. na face e T. ciliata no miolo, os quais apresentaram o melhor desempenho para essas propriedades.

Lima e Pio (2007) avaliaram outras espécies de folhosas de média densidade e encontraram valores para MOE e MOR paralelo, respectivamente, de 117.133,63 $\mathrm{kgf} / \mathrm{cm}^{2}$ e $773,18 \mathrm{kgf} / \mathrm{cm}^{2}$ para Copaifera duckei e de $102.027,32 \mathrm{kgf} / \mathrm{cm}^{2}$ e $701,10 \mathrm{kgf} / \mathrm{cm}^{2}$ para Eperua oleifera. Já para MOE e MOR perpendicular, os autores encontraram, respectivamente, $66.876,97 \mathrm{kgf} / \mathrm{cm}^{2}$ e $544,48 \mathrm{kgf} / \mathrm{cm}^{2}$ para Copaifera duckei e $74.239,09 \mathrm{kgf} /$ $\mathrm{cm}^{2}$ e $542,47 \mathrm{kgf} / \mathrm{cm}^{2}$ para Eperua oleifera. Com esses resultados, verifica-se que a resistência e a rigidez dos compensados de $T$. ciliata são inferiores em relação a painéis produzidos com outras folhosas apresentados na literatura, o que pode ser resultado da sua baixa densidade.

\subsubsection{Cisalhamento seco, úmido e pós-fervura}

As análises de variância realizadas para as propriedades de cisalhamento seco, cisalhamento úmido e cisalhamento pós-fervura mostraram que somente houve efeito significativo dos tratamentos sobre o primeiro. Os valores médios dessas variáveis encontram-se na Tabela 4.

Os dados da Tabela 4 mostram que o valor médio de resistência ao cisalhamento seco foi maior para o tratamento 6. Como a tensão de ruptura ao ensaio de cisalhamento foi realizada entre lâminas de Pinus sp., pode ter ocorrido aumento da resistência desses painéis a essa propriedade. Os menores valores médios foram encontrados para os tratamentos compostos somente por lâminas de Toona ciliata, os quais não apresentaram diferenças estatísticas significativas entre si. Segundo Marra (1992), a densidade e a porosidade da madeira têm influência na formação da linha de cola e ligação entre as lâminas que constituem a chapa. Assim, como a espécie Toona ciliata possui uma baixa densidade e alta porosidade, podem ter ocorrido linhas de cola famintas e, consequentemente, os painéis apresentaram baixa resistência ao cisalhamento independente da gramatura de cola e pressão. Além da porosidade, o alto teor de extrativos da madeira desta espécie também pode ter influenciado a baixa resistência da linha de cola.

De forma geral, os valores médios encontrados neste experimento para cisalhamento seco foram inferiores àqueles geralmente encontrados na literatura. Iwakiri et al. (2001) encontraram valores médios de resistência da linha de cola para painéis produzidos com resina fenol-formaldeído entre $23,38 \mathrm{kgf} / \mathrm{cm}^{2}$ (Pinus caribaea) e $35,67 \mathrm{kgf} / \mathrm{cm}^{2}$ (Pinus taeda) para teste seco. No entanto, todos os tratamentos apresentaram valores de tensão acima do valor mínimo de $10,2 \mathrm{kgf} / \mathrm{cm}^{2}$ exigido pela norma européia EN 314-2 (1993) a partir do qual não há exigências quanto à falha na madeira.

Tabela 4 - Valores médios de cisalhamento seco, úmido e pós-fervura e respectivas falhas na madeira.

Table 4 - Average values of the dry, humid and after boiling glue line shear strength and their wood failures.

\begin{tabular}{lcccccc}
\hline \multirow{2}{*}{ Tratamento } & \multicolumn{5}{c}{ Cisalhamento $\left(\mathrm{kgf} / \mathrm{cm}^{2}\right)$} \\
& $\mathrm{CS}$ & $\mathrm{FM}$ & $\mathrm{CU}$ & $\mathrm{FM}$ & $\mathrm{CF}$ & $\mathrm{FM}$ \\
\hline $1\left(\mathrm{~T}_{320 / 8}\right)$ & $16,80 \mathrm{~b}$ & $58,33 \mathrm{a}$ & $20,05 \mathrm{a}$ & $14,00 \mathrm{a}$ & $18,57 \mathrm{a}$ & $63,33 \mathrm{a}$ \\
$2\left(\mathrm{~T}_{320 / 5}\right)$ & $17,67 \mathrm{ab}$ & $54,67 \mathrm{a}$ & $18,54 \mathrm{a}$ & $19,33 \mathrm{a}$ & $21,05 \mathrm{a}$ & $34,67 \mathrm{a}$ \\
$3\left(\mathrm{~T}_{280 / 8}\right)$ & $17,70 \mathrm{ab}$ & $51,33 \mathrm{a}$ & $19,86 \mathrm{a}$ & $48,00 \mathrm{a}$ & $20,17 \mathrm{a}$ & $42,33 \mathrm{a}$ \\
$4\left(\mathrm{~T}_{280 / 5}\right)$ & $21,61 \mathrm{ab}$ & $74,00 \mathrm{a}$ & $22,23 \mathrm{a}$ & $48,00 \mathrm{a}$ & $20,50 \mathrm{a}$ & $70,00 \mathrm{a}$ \\
$5\left(\mathrm{P} / \mathrm{T}_{320 / 8}\right)$ & $22,6 \mathrm{ab}$ & $63,67 \mathrm{a}$ & $18,34 \mathrm{a}$ & $41,33 \mathrm{a}$ & $20,36 \mathrm{a}$ & $36,67 \mathrm{a}$ \\
$6\left(\mathrm{~T} / \mathrm{P}_{320 / 8}\right)$ & $23,88 \mathrm{a}$ & $59,67 \mathrm{a}$ & $19,67 \mathrm{a}$ & $20,00 \mathrm{a}$ & $20,23 \mathrm{a}$ & $24,67 \mathrm{a}$ \\
\hline F calculado & $4,968 *$ & $0,700 *$ & 0,554 ns & $4,337 *$ & $0,241 \mathrm{~ns}$ & $1,353^{\mathrm{ns}}$ \\
$\mathrm{CV}(\%)$ & 11,69 & 27,27 & 16,39 & 41,00 & 14,58 & 58,04 \\
\hline
\end{tabular}

T: Toona ciliata M. Roem.; P/T: Pinus sp. na face e Toona ciliata M. Roem. no miolo; T/P: Toona ciliata M. Roem. na face e Pinus sp. no miolo; gramatura de cola: 320 ou $280 \mathrm{~g} / \mathrm{m}^{2}$; pressão: 5 ou $8 \mathrm{kgf} / \mathrm{cm}^{2}$.

$\mathrm{e}^{*}$ : valores de F significativo a 5\% de significância; $\mathrm{e}^{\text {ns: }}$ valor de F não significativo a 5\% de significância.

Médias seguidas pela mesma letra não diferem estatisticamente entre si pelo teste de Tukey a $95 \%$ de probabilidade.

CV: Coeficiente de variação; FM: falha na madeira (\%), correspondente a cada teste de cisalhamento; CS: cisalhamento seco; CU: cisalhamento úmido; $\mathrm{CF}$ : cisalhamento pós-fervura.

Cerne, Lavras, v. 17, n. 1, p. 103-108, jan./mar. 2011 
Apesar dos valores médios de tensão para os ensaios de cisalhamento úmido e pós-fervura não terem sido significativos, como pode ser visto na Tabela 4, devese considerar que os mesmos estão acima do exigido pela norma européia EM 314-2 (valor mínimo de 10,2 kgf/ $\mathrm{cm}^{2}$ ), podendo ser indicados para o uso interior, intermediário e exterior (condição mais agressiva à linha de cola). De forma geral, os valores médios encontrados neste experimento para cisalhamento pós-fervura foram semelhantes àqueles geralmente encontrados na literatura. Normalmente, esperase que o adesivo fenólico resista à água, conservando seu estado físico e as fortes ligações químicas estabelecidas durante sua solidificação. Além de resistir à água, a resina fenólica, depois de solidificada, resiste à temperatura de fervura da água, podendo suportar temperaturas de até $200^{\circ} \mathrm{C}$ (HASELEIN; PAULESKI, 2004).

\section{CONCLUSÕES}

A madeira de Toona ciliata apresenta potencial para a produção de painéis compensados, tendo sido observados valores acima dos mínimos exigidos pelas normas correspondentes para todas as propriedades mecânicas analisadas, com exceção à flexão estática no sentido perpendicular.

Para os painéis produzidos somente com Toona ciliata, não houve efeito da gramatura de cola e da pressão sobre nenhuma das propriedades físicas e mecânicas analisadas.

A inclusão de lâminas de Pinus sp. nos painéis de Toona ciliata exerceu influência positiva sobre a maioria das propriedades físicas e mecânicas dos painéis.

\section{REFERÊNCIAS}

\section{ASSOCIAÇÃO BRASILEIRA DAS INDÚSTRIAS DE MADEIRA PROCESSADA MECANICAMENTE. Estudo setorial 2001. Curitiba, 2001. Disponível em: $<$ htpp://www. abimci.com.br>. Acesso em: 4 set. 2009.}

ASSOCIAÇÃO BRASILEIRA DE NORMAS TÉCNICAS. ABNT/CB-31: projeto de normas 31.000.05.001/1: chapas de madeira compensada. Rio de Janeiro, 2001.

BALDWIN, R. F. Plywood manufacturing practices. San Francisco: M. Freeman, 1995. 388 p.

EUROPEAN STANDARD-EN. Plywood: bonding quality: part 2: requirements. Cambridge, 1993.

Cerne, Lavras, v. 17, n. 1, p. 103-108, jan./mar. 2011
GUIMARÃES JÚNIOR, J. B.; MENDES, L. M.; MENDES, R. F.; MORI, F. A. Painéis compensado de eucalipto: estudo de caso de espécies e procedências. Cerne, Lavras, v. 15, n. 1, p. 10-18, 2009.

HASELEIN, C. R.; PAULESKI, D. T. Caderno didático da disciplina de Tecnologia da Madeira II, parte II. Santa Maria: UFSM, 2004. 53 p.

IWAKIRI, S. Painéis de madeira reconstituída. Curitiba: FUPEF, 2005. 254 p.

IWAKIRI, S.; OLANDOSKI, D. P.; LEONHARDT, G.; BRAND, M. A. Produção de chapas de madeira compensada de cinco espécies de pinus tropicais. Ciência Florestal, Santa Maria, v. 11, n. 2, p. 71-77, 2001.

IWAKIRI, S.; PRATA, J. G. Produção de painéis compensados multilaminados de Eucalyptus. Revista da Madeira, São Paulo, v. 111, p. 88-93, 2008.

IWAKIRI, S.; RAZERA NETO, A.; ALMEIDA, B. C.; BIASI, C. P.; CHIES, D.; GUISANTES, F. P.; FRANZONI, J. A.; RIGATTO, P. A.; BETTEGA, W. P. Avaliação da qualidade do compensado fenólico de Eucalyptus grandis. Ciência Florestal, Santa Maria, v. 16, n. 4, p. 437-443, 2006.

KOLLMANN, F. F. P.; KUENZI, E. W.; STAMM, A. J. Wood based materials. In: KOLLMANN, F. F. P. Principles of wood science and technology. Berlim: Springer-Verlag, 1975. v. 2, $703 \mathrm{p}$.

LAMPRECHT, H. Silvicultura nos trópicos. Eschborn: Gmbh, 1990. 343 p.

LIMA, N. N.; PIO, N. S. Avaliação da gramatura de cola na propriedade de flexão estática em painéis compensados de Copaifera duckei Dwayer e Eperua oleifera Ducke. Acta Amzonica, Manaus, v. 37, n. 3, p. 347-352, 2007.

MARRA, A. A. Technology of wood bonding: principles in practice. New York: V. N. Reinhold, 1992. 453 p.

PINHEIRO, A. L.; LANI, J. L.; COUTO, L. Cedro-

Australiano: cultivo e utilização (Toona ciliata M. Roem. var. australis (F. Muell) Bahadur). Viçosa, MG: UFV, 2006. 42 p.

SELLERS, T. Plywood and adhesive technology. New York: M. Dekker, 1985. 661 p. 